\title{
Cardiac Function Evaluation with Cine MRI of the Heart
}

MRI is considered to be the gold standard for the calculation of hemodynamic parameters of cardiac function such as ejection fraction (EF), end diastolic volume (EDV), end systolic volume (ESV), stroke volume (SV), and cardiac mass. With MRI, cine loops of the heart are acquired over several heartbeats in order to perform enough image encoding steps for each cardiac phase. The acquisition of image data for each cardiac phase is synchronized with the heart cycle by gating of the encoding steps with the patients electrocardiogram (ECG). Recently, it has also become feasible to acquire cine loops in real time, although the temporal resolution is not optimal, even with current state-of-theart MR scanners. Nevertheless, in patients without regular rhythm, these new real-time techniques have started to offer an acceptable alternative.

Despite the recognized strengths of cardiac MRI for the assessment of cardiac function, its use still lags far behind the use of echocardiography. Besides issues of cost and availability of dedicated cardiovascular MRI scanners, the lack of training in this area may also have contributed to a delay in adopting cardiac MRI for cardiac function evaluation. This unit is meant as a practical guide to some useful MRI protocols for cardiac function evaluation. The parameters of these protocols are based on experience on a Siemens 1.5 T Sonata scanner and should be altered accordingly for different field strengths and manufacturers.

Imaging of cardiac function is currently best performed with cardiac-gated pulse sequences that split the image acquisition for each frame into a number of segments that are acquired over, typically, 10 to 20 heartbeat cycles. Still, there are many options to consider when doing these functional studies: breath hold versus free breathing; prospective triggering versus retrospective gating; and volumetric data sets versus biplanar approaches. Other issues that factor in are the patient's heart rate or rhythm, degree of functional impairment, the presence of valvular disease, and the need to assess for jets from shunts or valve dysfunction. There is no one unique way to do these studies, as trade-offs must be made to accommodate a particular clinical situation.

The sharpest images are obtainable with breath holding and use of a segmented $k$-space approach (Schulen et al., 1996). The more image encoding steps (i.e., lines of $k$-space) acquired per heartbeat, the shorter the necessary breath-hold duration. However, temporal resolution per cardiac phase will be reduced and there will be loss of myocardial-bloodpool contrast because of saturation effects, noticeable in particular for later phases of the cardiac cycle. The effective temporal resolution can be improved using view-sharing interpolation, whereby some image-encoding steps are shared for the reconstruction of images for adjacent cardiac phases. It is best to have an effective temporal resolution of $\leq 40 \mathrm{msec}$, particularly for rapid heart rates.

Gating of the image acquisition can be performed prospectively. The repetition of the encoding steps in the pulse sequence is then controlled by a physiologic marker, e.g., the peak of the $R$-wave in the electrocardiogram. (The $R$-wave peak in the electrocardiogram coincides with the closing of the mitral valve, i.e., with the end of diastole.) By contrast, with retrospective gating, the pulse sequence continuously acquires data, and the physiological gating signal is recorded independently and is not used to time the image acquisition. During image reconstruction, each acquired phase encoding is assigned to a phase of the cardiac cycle according to the physiological marker. Retrospectively gated cardiac cine sequences require some redundancy in the acquired data to assure that all 


\begin{tabular}{ll}
\hline Coil type & Quadrature phase array torso coil \\
Gradient coil strength & $24 \mathrm{mT} / \mathrm{m}$ (or higher if the system permits) \\
Cardiac gating & Yes \\
Peripheral gating & For monitoring only \\
Respiratory gating & No \\
Respirator & If required by patient \\
Oxygen & If required by patient \\
Breath-holding & Optional \\
Motion cushions & Can be used for patient comfort \\
Use of contrast agents & No \\
Infusion pump & Required for pharmacological stress \\
Monitoring equipment & Heart rate, oxygen saturation, and blood \\
& pressure should be monitored with \\
& MRI-compatible equipment \\
\hline
\end{tabular}

phases of the cardiac cycle have been sampled adequately for image reconstruction. Retrospective gating allows for coverage of the entire cardiac cycle. Some manufacturers allow for rejection of data acquired from premature ventricular contractions with their retrospective gating software. It is not possible, however, to obtain the data in one breath-hold duration, as these pulse sequences tend to be lengthier than prospectivelygated acquisitions. Generally, prospective triggering is adequate. However, it is important to obtain as many phases as possible without spilling into the next cardiac cycle. Allowances must be made for change in heart rate during the data acquisition. Usually, it is sufficient to cover less than the R-to-R interval by for example, $100 \mathrm{msec}$ of the cardiac cycle. The entire cine protocol with acquisition of localizers for planning of cine acquisitions takes $\sim 15 \mathrm{~min}$.

Table A11.4.1 lists the hardware necessary to perform cardiac function evaluation with cine MRI.

NOTE: Be sure the technologists and nurses have immediate access to any emergency equipment that may be relevant to a given study, or that may be needed for a particular patient such as crash carts or oxygen.

\section{Set up patient and equipment}

1. Take standard precautions as with any other MRI exam when preparing the patient for an MRI examination of cardiac function. Check for possible counter-indications and for ferromagnetic implants. For patients with heart disease, monitor heart rate, blood pressure, and other vital signs while the patient undergoes the MRI examination. Emergency equipment such as a defibrillator, crash cart, and emergency medications for treating cardiac arrests (e.g., epinephrine) should be nearby.

Generally, standard screening forms (see APPENDIX 1) are used for all patients scanned in a magnetic resonance system.

In patients with ferromagnetic implants, if in doubt as to the exact composition of the items, it is best to exclude patients with any metal implants; see Shellock (1996) for a discussion of what implants may be safely scanned using magnetic resonance.

Patients may be accompanied into the magnet room by a friend or family member, who can sit in the room during the scan and comfort the patient as needed. This companion must be screened as well to ensure the absence of loose metal objects on the body or clothing.

Cardiac Function
Evaluation with
Cine MRI of the
Heart

A11.4.2 
2. If the procedure is a research protocol, have the patient sign any necessary consent forms.

3. Have the patient remove all jewelry and change into a gown to eliminate any metal that might be found in clothing.

4. Have the patient wash off any mascara and other makeup to avoid local tissue heating.

5. Explain to the patient the image protocol and what he or she should expect during the exam:

a. Ascertain whether the patient can hold his/her breath for $\sim 10$ to $15 \mathrm{sec}$.

b. Provide the patient with ear plugs or headphones and let the patient know, in advance, that the pulse sequences will cause a loud noise in the magnet. Instruct the patient on how he/she can communicate with the person controlling the MRI scanner during the entire duration of the scan.

c. Instruct the patient not to move and to maintain a regular breathing pattern while he or she hears the scanner acquiring images.

d. Nevertheless, the patient may call out at any time if he or she feels it necessary.

6. Position the patient supine on the patient bed. Provide the patient with a cushion under the legs for comfort.

7. Place the ECG electrodes around the heart with $\leq 2$ to 3 in. of separation between them. This results in an improved quality of the ECG signal and more reliable triggering of the pulse sequence. Align the ECG wires parallel to the magnetic flux lines of the static magnetic field. Conductive cables should not cross inside the bore, and they should also be kept away from the sides of the bore. These precautions are necessary to minimize the risk of burns due to radio frequency power deposition.

Optimal ECG triggering is vital to obtaining a quality MRI examination. Nevertheless, optimal ECG triggering is often problematic in cardiac MRI examinations due to degradation of the ECG signal by superimposed voltages that are generated by blood flow through the great vessels while the patient is positioned in a strong magnetic field. Special MR-compatible physiologic recorders, which can be interfaced with the MRI scanner, often use customized ECG electrodes and proprietary filtering techniques to reduce artifacts in the ECG.

The ECG electrodes may be placed on the chest or back. Placing them on the chest is best for monitoring, but in this position the ECG signal is more susceptible to artifacts from breathing motion, as well as image artifacts from the leads affecting the right ventricle. The dry rub method improves electrode contact to the skin and reduces contact resistance. If the baseline of the ECG moves up and down with respiration, then the ECG leads need to be moved up higher on the chest. Asking the patient to avoid a deep breathing motion can help. Reducing the distance between the ECG leads can reduce interference from switching of the gradient coils.

The dry-rub method consists in the use of a slightly abrasive material, such as a clean, dry gauze pad, and rubbing it on the skin to remove skin oils and perspiration. This should allow proper adhesion of the ECG electrodes to the skin, which is essential for consistent ECG readings during the MRI study. The use of standard alcohol wipes may be necessary to remove an excess of oils, lotions, or perspiration.

8. Wrap a blood pressure cuff around the patient's arm to monitor the blood pressure. MRI-compatible monitoring systems use a pump that inflates and deflates the blood pressure cuff at predefined time intervals or when the technologist manually initiates a blood pressure measurement. Place a fiber-optic sensor on the patient's finger to measure the arterial oxygen saturation. Take note of the baseline heart rate, systemic blood pressure, and oxygen saturation. Repeat these measurements regularly during examination.

Acquired Heart

Disease

A11.4.3 
Table A11.4.2 Primary Clinical Imaging Parameters for Sequence 1 (Pilot Scan)

\begin{tabular}{|c|c|}
\hline Patient position & Supine \\
\hline Scan type & $\begin{array}{l}\text { Short } \mathrm{T}_{R} \text { gradient echo with } \\
\text { steady-state free precession. }\end{array}$ \\
\hline Imaging plane (orientation) & $\begin{array}{l}\text { Transverse and coronal, pseudo-long } \\
\text { axis, oblique short axis, or long axis } \\
\text { (four chamber) }\end{array}$ \\
\hline Central slice or volume center & Laser light centered on coil center \\
\hline Echo time $\left(T_{\mathrm{E}}\right)$ & $2.3 \mathrm{msec}$ \\
\hline Repeat time $\left(T_{\mathrm{R}}\right)$ & $5 \mathrm{msec}$ \\
\hline Flip angle (FA) & $50^{\circ}$ \\
\hline Fields of view $\left(\mathrm{FOV}_{\mathrm{x}}, \mathrm{FOV}_{\mathrm{y}}\right)$ & $400 \mathrm{~mm}, 340-400 \mathrm{~mm}$ \\
\hline Resolution $(\Delta x, \Delta y)$ & $1.56 \mathrm{~mm}, \sim 2.43-2.86 \mathrm{~mm}$ \\
\hline Number of data points collected $\left(N_{\mathrm{x}}, N_{\mathrm{y}}\right)$ & $256, \sim 140$ \\
\hline Display matrix $\left(D_{\mathrm{x}}, D_{\mathrm{y}}\right)$ & 256,256 \\
\hline Slice thickness $(\Delta z)$ & $8 \mathrm{~mm}$ \\
\hline Number of slices & $\begin{array}{l}3 \text { for transverse and } 3 \text { for coronal } \\
\text { views, } 3 \text { for pseudo-long axis views, } \\
5-6 \text { for oblique short-axis views, or } 1 \\
\text { for four-chamber view }\end{array}$ \\
\hline Slice gap & $\leq 2 \mathrm{~mm}$ \\
\hline Number of acquisitions $\left(N_{\text {acq }}\right)$ & 1 \\
\hline Swap read and phase encoding & $\begin{array}{l}\text { Swap if necessary (phase encoding } \\
\text { direction is anterior-posterior) }\end{array}$ \\
\hline Slice locations & Not applicable \\
\hline Saturation pulses & Not applicable \\
\hline ECG gating & Yes \\
\hline Scan time & $\begin{array}{l}\sim 1 \mathrm{sec} / \text { slice, total time is } \sim 6 \mathrm{sec} \\
\text { (transverse and coronal), } \sim 3 \mathrm{sec} \\
\text { (pseudo-long axis), or } 5-6 \mathrm{sec} \text { (oblique } \\
\text { short axis), or } \sim 1 \mathrm{sec} \text { (long axis, } \\
\text { four-chamber) }\end{array}$ \\
\hline
\end{tabular}

9. Use a dedicated cardiac coil for these studies to maximize the achievable signal-tonoise ratio. Center the coil at the level of the heart, or over the patient's heart, if a relatively small surface coil is used.

10. Instruct the patient on the procedure for breath holding during the MRI examination. Determine whether the patient is able to hold his/her breath for $\sim 10$ to $15 \mathrm{sec}$ at a time by practicing breath holding with the patient. When patient preparations have been finished, position the patient bed such that the centering light is aligned over the patient's heart, then move the table to have the patient's heart in the magnet center.

11. Patients with claustrophobia may require sedation to tolerate the procedure. If this is the case, follow institutional guidelines for conscious sedation.

\section{Sequence 1: Scout imaging of the heart}

12. Acquire a multi-plane scout (producing 3 transverse and 3 coronal slices) with a short $T_{\mathrm{R}}$ gradient echo sequence, preferably with steady-state free precession to obtain optimal signal-to-noise ratio. Typical sequence parameters for such a pulse sequence are listed in Table A11.4.2 and the resulting images are shown in Figure A11.4.1. Ask the patient to take a breath in and then hold his/her breath at end-expiration. Obtain transverse and coronal scout images using sequence parameters listed in Table A11.4.2. 
A

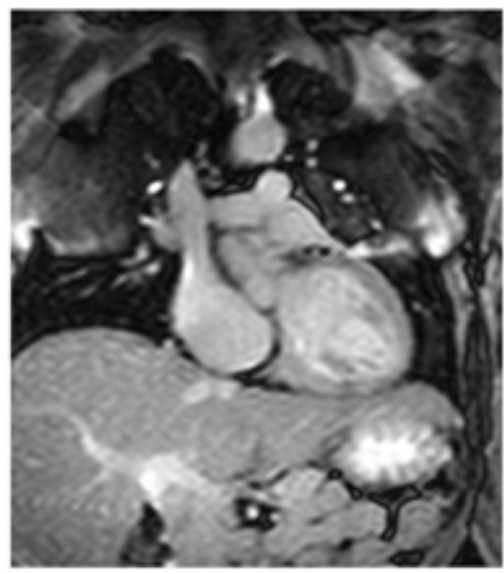

B

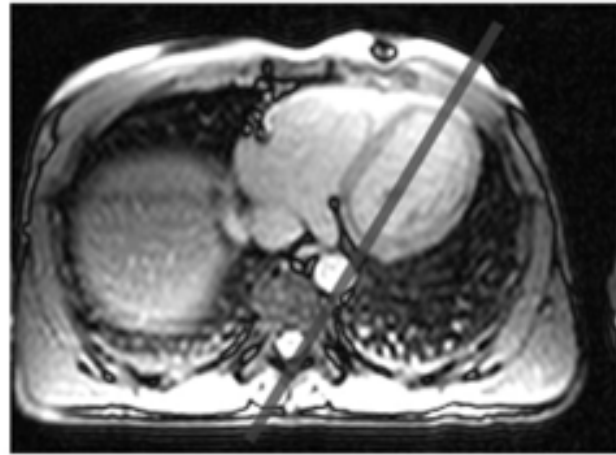

Figure A11.4.1 Coronal (A) and transverse (B) scout images acquired with single-shot steady-state free precession imaging technique while the patient holds his or her breath. The transverse scout image is used to position the slice for the pseudo-long-axis localizer. The slice position and orientation are shown in the image on $\mathbf{B}$ as a dark gray bar.

A

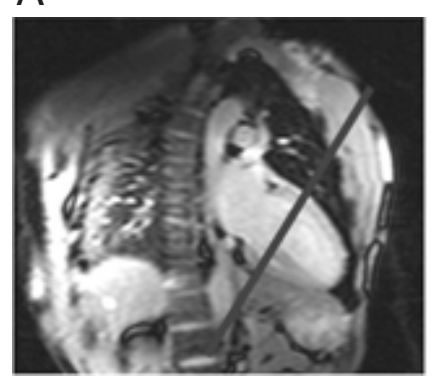

B

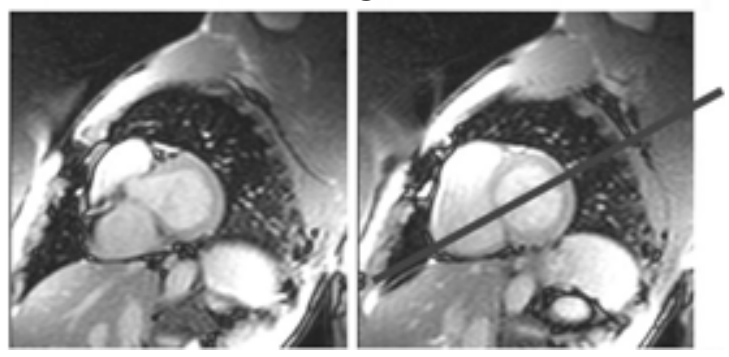

Figure A11.4.2 The image in A shows a pseudo-long vertical long-axis view of the heart. Use this scout image to position the slices for a series of short-axis views near the base of the heart. The resulting short-axis views are shown in images $\mathbf{B}$ and $\mathbf{C}$. The slice orientation for cine acquisition in a four-chamber, long-axis view should pass through the center of the left ventricle, and intersect with the antero-lateral tip of the right ventricle, as shown by the dark bar in image $\mathbf{C}$.

\section{Oblique pseudo-long-axis scout}

13. Prescribe on a transverse view through the middle of the left ventricle and acquire an image for this view as a localizer as shown in Figure A11.4.1. Ask the patient to take a breath in and then hold his/her breath at end-expiration. Obtain images using the same sequence parameters as in Table A11.4.2 with the orientation set to be the pseudo-long axis, as shown by the dark bar in Figure A11.4.1B. An example of the image obtained with this slice orientation appears in Figure A11.4.2A.

\section{Oblique short-axis scout}

14. Prescribe on the resulting pseudo-long-axis view in order to obtain a set of doubleoblique short-axis views near the base of the heart. Select the short-axis slice just below the valve plane (Fig. A11.4.2A) and prescribe a slice that passes through the middle of the left ventricle ( $\mathrm{LV}$ ) and intersects with the antero-laberal tip of the right ventricle. Ask the patient to take a breath in and then hold his/her breath at end-ex-

Acquired Heart

Disease

A11.4.5 


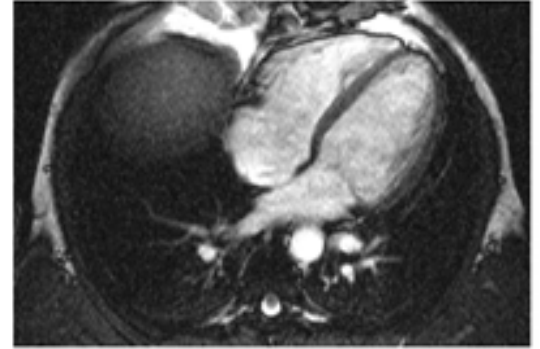

End diastole

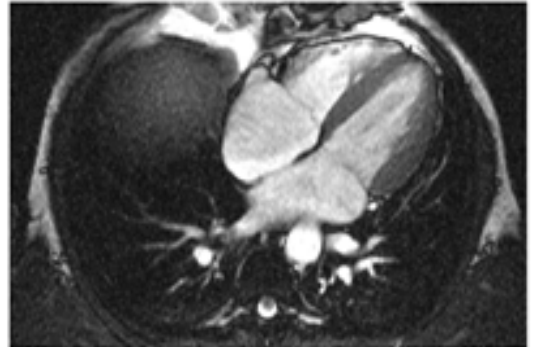

End systole

Figure A11.4.3 Two frames from a cine acquisition with steady state free precession sequence for a four-chamber long-axis view of the heart with the long-axis slice orientation prescribed as shown in Figure A11.4.2C.

piration. Obtain images (Fig. A11.4.2B and A11.4.2C) using parameters listed in Table A11.4.2 with orientation set to oblique short axis.

\section{Four-chamber view}

15. Prescribe a slice as indicated in Figure A11.4.2C. Obtain images using the parameters listed in Table A11.4.2 with orientation set to be the long axis four-chamber view (same orientation as shown in Fig. A11.4.3). The resulting image gives a long-axis four-chamber view of the hear.

\section{Sequence 2: Segmented cine imaging}

For patients with cardiac arrhythmias, one might have to resort to a real-time imaging sequence. This means that the acquisition of each cine frame is no longer broken up into segments, but rather acquired in one single shot. Often, the real-time imaging techniques require extensive interpolation of imaging data from adjacent cardiac phases to produce a smooth cine loop. For details about setting up the real-time acquisition, see sequence 3 .

16. The authors recommend that a cine of a long-axis, four-chamber view (horizontal long axis) be acquired first (Fig. A11.4.3). For this purpose, copy the slice position and orientation of the scout four-chamber view obtained with sequence 1 in step 15.

In the long-axis view, the slice orientation results in the less flow-driven enhancement of the signal intensity in the ventricular cavity. This can be countered by choosing a thinner slice thickness.

17. Adjust the sequence parameters with the orientation set to be the double-oblique long (four-chamber) axis (either Table A11.4.3 or Table A11.4.4; depending on the capabilities of the scanner and the patient's heart rate). Acquire a minimum of 15 cardiac phases for either cine loop. Adjust the number of phase-encoding lines per cardiac phase correspondingly.

This setting is also referred to as the number of lines per segment, in reference to the fact that the acquisition of the images is broken up into segments and for each segment, a number of $k$-space lines is acquired. For example, for a $T_{R}=7 \mathrm{msec}$ per phase-encoding step and a heart rate of 70 beats/min, one should allot 40 to 50 msec to each cardiac phase, and with a $T_{R}=7$ msec, $\sim 5$ to 7 lines (i.e., 40/7 to 50/7) can be acquired per segment.

The optimal flip angle that produces the best contrast on cine images between the myocardium and the LV chamber depends, among other parameters, on the slice thickness and the repetition time $\left(T_{R}\right)$. It is worthwhile to gather some initial experience with cine $M R$ imaging of the heart in a volunteer to optimize the flip angle and other sequence parameters. It is impossible to recommend fixed settings for all pulse sequence parameters for users of MRI platforms from different vendors. The overall goal of this exam should be 
Table A11.4.3 Primary Clinical Imaging Parameters for Sequence 2 (Steady-State Free Precession Cine Imaging)

\begin{tabular}{|c|c|}
\hline Patient position & Supine \\
\hline Scan type & $\begin{array}{l}\text { Segmented } k \text {-space cine gradient echo } \\
\text { with view sharing }\end{array}$ \\
\hline Imaging plane (orientation) & $\begin{array}{l}\text { Double oblique, short- or or long- } \\
\text { (four-chamber) axis view }\end{array}$ \\
\hline Central slice or volume center & Mid-level of left ventricle \\
\hline Echo time $\left(T_{\mathrm{E}}\right)$ & $1.5-1.6 \mathrm{msec}$ \\
\hline Receiver bandwidth (RBW) & 238 kHz ( 930 Hz/pixel) \\
\hline Number of lines per segment & 9 \\
\hline Repeat time $\left(T_{\mathrm{R}}\right)$ & $\begin{array}{l}27 \mathrm{msec}\left(T_{\mathrm{R}} \text { per } k \text {-space segment, }\right. \\
\text { temporal resolution) }\end{array}$ \\
\hline Delay time $\left(T_{\mathrm{D}}\right)$ & $0 \mathrm{msec}$ \\
\hline Flip angle (FA) & $60^{\circ}$ \\
\hline Fields of view $\left(\mathrm{FOV}_{\mathrm{x}}, \mathrm{FOV}_{\mathrm{y}}\right)$ & $\begin{array}{l}300-360 \mathrm{~mm}, 300 r-360 r \mathrm{~mm} \text {, with } r \\
\leq 0.75 \text { (rectangular field of view), } \\
\text { depending on body habitus }\end{array}$ \\
\hline Resolution $(\Delta x, \Delta y)$ & $1.17-1.41 \mathrm{~mm}, 2.17-2.61 \mathrm{~mm}$ \\
\hline Number of data points collected $\left(N_{\mathrm{x}}, N_{\mathrm{y}}\right)$ & $\begin{array}{l}256,138 r \text {, with } r \leq 3 / 4 \text { (rectangular } \\
\text { field of view) depending on body } \\
\text { habitus }\end{array}$ \\
\hline Display matrix $\left(D_{\mathrm{x}}, D_{\mathrm{y}}\right)$ & 256,256 \\
\hline Slice thickness $(\Delta z)$ & $6-8 \mathrm{~mm}$ \\
\hline Number of slices & 1 \\
\hline Slice gap & N/A \\
\hline Number of acquisitions $\left(N_{\mathrm{acq}}\right)$ & 1 \\
\hline Swap read and phase encoding & Swap if wrap-around is reduced \\
\hline Slice location & $\begin{array}{l}\text { Long axis of heart from mitral valve } \\
\text { center to apical tip (the heart is } \\
\text { covered from base to apex for the } \\
\text { short-axis view) }\end{array}$ \\
\hline Saturation pulses & No \\
\hline Number of cardiac phases & $(R$-to- $R$ interval $) \times 90 \% / T_{\mathrm{R}}$ (at least 15$)$ \\
\hline ECG gating & Yes \\
\hline Scan time & $\sim 9 \mathrm{sec}$ \\
\hline
\end{tabular}

to (a) assure complete coverage of the cardiac cycle by adjustment of the number of cardiac phases, (b) good image quality that provides excellent contrast at the endocardial border, and (c) complete coverage of the heart from base to apex. While image quality depends on some aspects not entirely under the control of the MRI operator such as patient cooperation, complete temporal and spatial resolution is the operator's responsibility. Failure to meet these requirements can render the acquired data useless for LV function analysis.

18. Ask the patient to take a breath in and then hold his/her breath at end-expiration. Start the scan after the patient has begun to hold his/her breath (Fig. A11.4.4).

Breath-holding at end-expiration seems to result in a more reproducible diaphragm position, than if the patient stops breathing at end-inspiration.

19. During the acquisition of the images, one should listen to the noise from the switching gradients and make sure that the noise follows the cardiac rhythm. Do not forget to tell the patient when he or she can start to breathe again, as any negligence or lack of courtesy may result in less cooperation from the patient.

Acquired Heart

Disease

A11.4.7 
Table A11.4.4 Primary Clinical Imaging Parameters for Sequence 2 (Bright-Blood Cine Gradient Echo)

\begin{tabular}{|c|c|}
\hline Patient position & Supine \\
\hline Scan type & Segmented $k$-space cine gradient echo \\
\hline Imaging plane (orientation) & $\begin{array}{l}\text { Double oblique, long- (four-chamber), } \\
\text { or short-axis view }\end{array}$ \\
\hline Central slice or volume center & Mid-level of left ventricle \\
\hline Echo time $\left(T_{\mathrm{E}}\right)$ & $\begin{array}{l}4.8 \mathrm{msec} \text { with flow compensation } \\
2.7 \mathrm{msec} \text { without flow compensation }\end{array}$ \\
\hline Receiver bandwidth (RBW) & $50 \mathrm{kHz}(\sim 197 \mathrm{~Hz} /$ pixel $)$ \\
\hline Number of lines per segment & $5-7$ \\
\hline Repeat time $\left(T_{\mathrm{R}}\right)$ & 40-56 msec (temporal resolution) \\
\hline Delay time $\left(T_{\mathrm{D}}\right)$ & $0 \mathrm{msec}$ \\
\hline Flip angle (FA) & $20^{\circ}$ \\
\hline Fields of view $\left(\mathrm{FOV}_{x}, \mathrm{FOV}_{y}\right)$ & $\begin{array}{l}300-360 \mathrm{~mm}, 300 r-360 r \mathrm{~mm}, \mathrm{r}=3 / 4 \\
\text { (rectangular field of view) depending } \\
\text { on body habitus }\end{array}$ \\
\hline Resolution $(\Delta x, \Delta y)$ & $1.17-1.41 \mathrm{~mm}, 2.21-2.65 \mathrm{~mm}$ \\
\hline Number of data points collected $\left(N_{\mathrm{x}}, N_{\mathrm{y}}\right)$ & $\begin{array}{l}256,136 r \text {, with } \mathrm{r}=3 / 4 \text { (rectangular } \\
\text { field of view) depending on body } \\
\text { habitus }\end{array}$ \\
\hline Display matrix $\left(D_{\mathrm{x}}, D_{\mathrm{y}}\right)$ & 256,256 \\
\hline Slice thickness $(\Delta z)$ & $6-8 \mathrm{~mm}$ \\
\hline Number of slices & 1 \\
\hline Slice gap & N/A \\
\hline Number of acquisitions $\left(N_{\text {acq }}\right)$ & $\begin{array}{l}1 \text { with breath-holding, } 3 \text { without } \\
\text { breath-holding }\end{array}$ \\
\hline Swap read and phase encoding & Swap if wrap around is reduced \\
\hline Slice location & $\begin{array}{l}\text { Long axis of heart from mitral valve } \\
\text { center to apical tip (the heart is } \\
\text { covered from base to apex for the } \\
\text { short-axis view) }\end{array}$ \\
\hline Saturation pulses & No \\
\hline Number of cardiac phases & $(R$-to- $R$ interval $) \times 90 \% / T_{\mathrm{R}}$ (at least 15$)$ \\
\hline ECG gating & Yes \\
\hline Scan time & $<17 \mathrm{sec}$ \\
\hline
\end{tabular}

Irregular gradient noise occurs when the patient has an irregular cardiac rhythm or if the acquisition window is very close to the $R$-to- $R$ duration. In the latter case, the very slight variations in heart rate, e.g., due to breath-holding, cause the sequence to be triggered randomly every heartbeat or every second heartbeat. Irregular triggering prevents establishment of a steady state and results in ghosting artifacts.

Fat saturation requires a very good shim to prevent inadvertent suppression of water signal. Generally, there is little benefit in the use of fat saturation when the objective is obtaining functional data.

20. After acquisition of a set of images for a slice position, play all cardiac phases as a cine loop and make sure that image quality is sufficient and that the cine loop covers the cardiac cycle as required. Repeat the acquisition if necessary, or otherwise move to the next slice position.

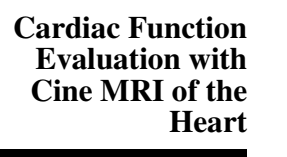

A11.4.8

Ghosting artifacts propagating along the phase-encoding direction are a tell-tale sign of breathing-induced motion. The acquisition of these cine-loops should be repeated if ghosting is found. 


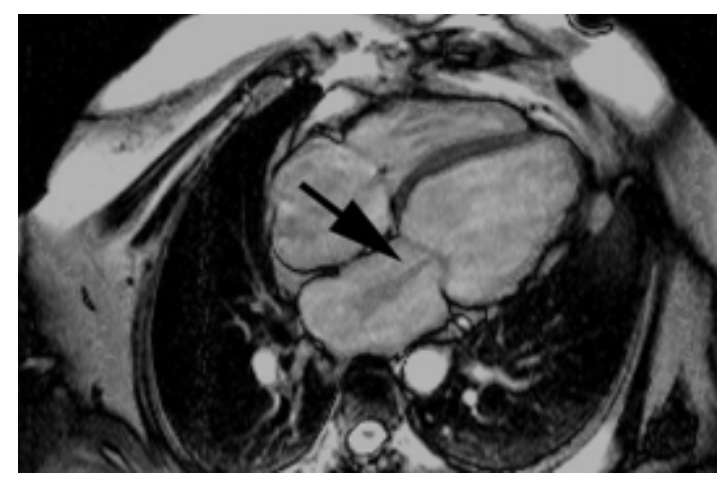

Figure A11.4.4 Regurgitant jet (highlighted by the arrow) in a patient with myocardial infarction and acute mitral valve regurgitation that developed with severe ventricular failure. The cine images were acquired with a steady-state free precession (SSFP) sequence. With SSFP sequences, turbulent flow causes pronounced signal loss and is well visualized.

21. If it seems that the patient is uncooperative or simply unable to hold his/her breath, attempt to reduce the required breath-hold duration or switch to a nonbreath-hold acquisition. To reduce the breath-hold duration, increase the number of phase encoding steps per cardiac phase (i.e., number of lines per segment). This will reduce the total number of cardiac phases in the cine loop, i.e., it reduces temporal resolution. Try to further reduce the field of view, as long as the aliasing (wrap-around artifact) does not obstruct view of the heart. For non-breath-hold acquisitions, all image parameters, except the number of acquisitions, can be left unchanged. To average out breathing motion, average at least 3 acquisitions. Ask patient to breathe regularly.

22. Obtain short-axis images by positioning the acquisition off the diastolic frame of the horizontal long axis (four-chambered long axis) cine image. The heart should be covered from base to apex, starting at a position above the mitral valve plane. Adjust the position such that the slice position is a number that is easy to increment. For example, instead of a slice position of $83.4 \mathrm{~mm}$, use a slice position of $80 \mathrm{~mm}$ with 8 -mm increments to cover the heart with consecutive breath holds. In cases of low signal-to-noise ratio, choose a thicker slice for short axis images than for long axis images (e.g., $8 \mathrm{~mm}$ for short axis images versus $6 \mathrm{~mm}$ for the long axis images).

23. Ask the patient to hold his/her breath. To obtain the first slice, run sequence 2 according to either Table A11.4.3 or Table A11.4.4, with the orientation set to be the doubleoblique short axis. Ask the patient to breathe and hold his/her breath again. Run sequence 2 according to either Table A11.4.3 or Table A11.4.4 to obtain the second slice. Repeat this procedure until the entire heart is covered (from base to apex).

To assure complete coverage of the heart, the short axis cine acquisitions should be repeated until one reaches the apical tip of the heart. No bright signal from the ventricular blood pool should be visible in any of the cine frames for the most apical slice location.

The sum of slice thickness and the slice gap between adjacent slices should be equal to the slice increment, e.g., if the slice increment is $8 \mathrm{~mm}$ and the slice thickness is $6 \mathrm{~mm}$, then the resulting slice gap between adjacent slices is $2 \mathrm{~mm}$. 
Table A11.4.5 Primary Clinical Imaging Parameters for Sequence 3 (Phase-Contrast Cine of Aorta)

\begin{tabular}{|c|c|}
\hline Patient position & Supine \\
\hline Scan type & $\begin{array}{l}\text { Fast gradient echo with phase-contrast } \\
\text { flow velocity encoding }\end{array}$ \\
\hline Imaging plane (orientation) & $\begin{array}{l}\text { Close to transverse orientation, } \\
\text { perpendicular intersection with } \\
\text { ascending aorta }\end{array}$ \\
\hline Central slice or volume center & $\begin{array}{l}\text { At level of bifurcation of pulmonary } \\
\text { trunk }\end{array}$ \\
\hline Echo time $\left(T_{\mathrm{E}}\right)$ & $3.6 \mathrm{msec}$ \\
\hline Repeat time $\left(T_{\mathrm{R}}\right)$ & 20 msec (temporal resolution) \\
\hline Flip angle (FA) & $15^{\circ}$ \\
\hline Fields of view $\left(\mathrm{FOV}_{\mathrm{x}}, \mathrm{FOV}_{\mathrm{y}}\right)$ & $300 \mathrm{~mm}, 244 \mathrm{~mm}$ \\
\hline Resolution $(\Delta x, \Delta y)$ & $1.17 \mathrm{~mm}, 1.89 \mathrm{~mm}$ \\
\hline Number of data points collected $\left(N_{\mathrm{x}}, N_{\mathrm{y}}\right)$ & $256, \sim 129$ \\
\hline Display matrix $\left(D_{\mathrm{x}}, D_{\mathrm{y}}\right)$ & 256,256 \\
\hline Slice thickness $(\Delta z)$ & $5 \mathrm{~mm}$ \\
\hline Number of slices & 1 \\
\hline Slice gap & Not applicable \\
\hline Number of acquisitions $\left(N_{\text {acq }}\right)$ & 2 \\
\hline Swap read and phase encoding & $\begin{array}{l}\text { Swap if necessary for } \\
\text { anterior-posterior as the phase } \\
\text { encoding direction }\end{array}$ \\
\hline Slice locations & Ascending aorta \\
\hline Saturation pulses & Not applicable \\
\hline Number of cardiac phases & Between 25 and 35 \\
\hline Vascular options & $\begin{array}{l}\text { Velocity encoding sensitivity of } \pm 150 \\
\mathrm{~cm} / \mathrm{sec}\end{array}$ \\
\hline Scan time & $\begin{array}{l}\sim 129 \text { heartbeats per acquisition (with } \\
129 \text { phase-encoding steps and } 25-35 \\
\text { cardiac phases). The total scan time is } \\
\sim 258 \text { heartbeats. }\end{array}$ \\
\hline
\end{tabular}

\section{Sequence 3: Phase-contrast flow velocity imaging of aorta}

In the presence of aortic regurgitation, the stroke volumes calculated by volumetric analysis will be different from the effective (forward) flow volume. See Commentary for volumetric analysis. For determination of the effective stroke volume, it then becomes necessary to supplement these measurements with phase-contrast flow velocity measurements, using sequence 3 .

24. Load sequence 3 (Table A11.4.5) on the console. Position the slice at the level of the bifurcation of the pulmonary trunk (Fig. A11.4.5). Intersect the slice with the ascending aorta at a right angle for accurate quantification blood flow. Enable the reconstruction of phase and magnitude images. The phase images provide the velocity information.

Sequence 3 should be loaded with through-plane flow quantification, which means that flow is encoded for a direction perpendicular to the slice plane.

For through-plane flow encoding, a tilt of the image plane from an orientation orthogonal to the vessel axis by an angle $\alpha$ will result in a reduction of the flow velocity estimate below the true flow velocity by a factor $\cos (\alpha)$. 
A

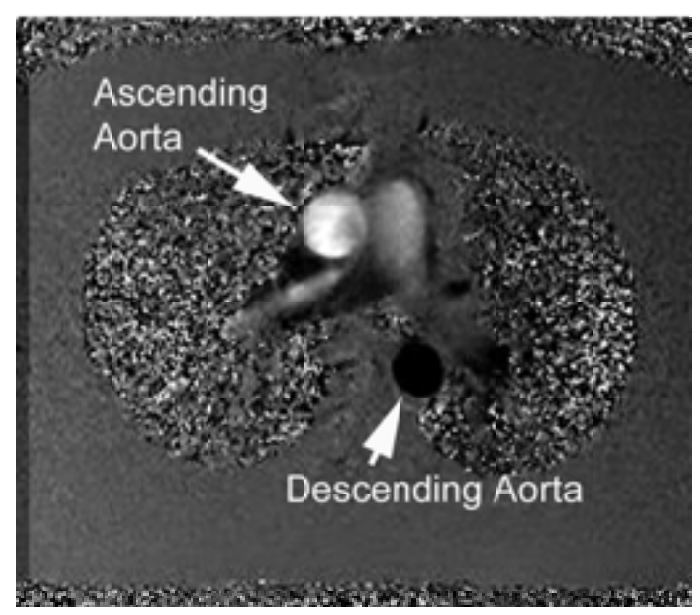

B

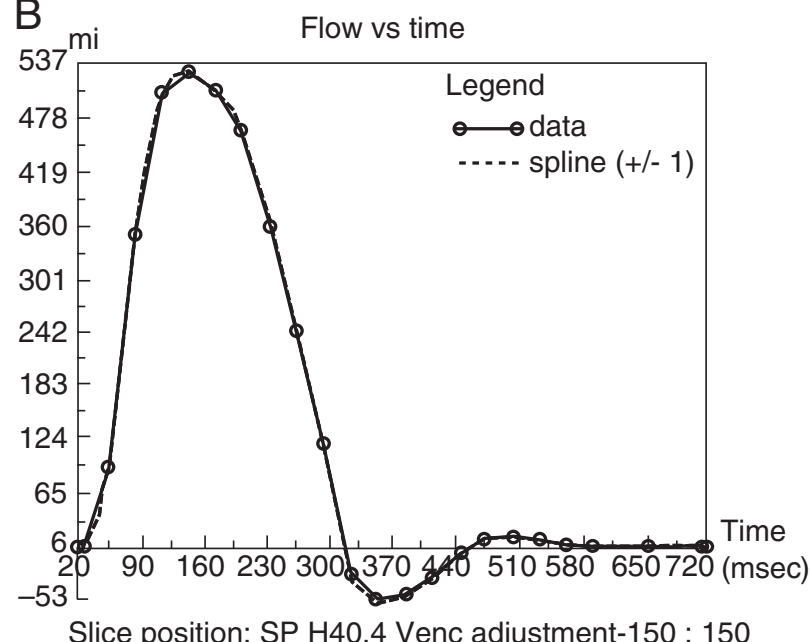

Figure A11.4.5 Panel A shows an image of the signal phase (after background correction) in a patient at the level of bifurcation of the pulmonary trunk. Flow was encoded in a direction perpendicular to the slice plane. The slice plane was oriented to intersect the ascending aorta at a right angle. The phase of the signal is encoded on a gray scale, and the phase is directly proportional to the flow velocity. Panel B shows a graph of aortic flow versus the delay time after the $R$-wave. The flow is calculated from the phase images by integrating the flow velocity over the aortic cross-section to obtain flow in units of $\mathrm{ml} / \mathrm{sec}$, in this case. Flow analysis software is available as an option on most MRI scanners.

25. Adjust the number of cardiac phases to anywhere between 25 and 35, depending on the patient's heart rate. Perform a magnetic field shim before running the sequence, as magnetic field inhomogeneities primarily alter the phase of the signal, and can cause erroneous flow velocity estimates.

26. It is generally not possible to complete the flow velocity measurement within one breath-hold. Instruct the patient to breathe normally, and use two averages (2 acquisitions or excitations) to average out the breathing motion. Inform the patient that the total scan time will take $\sim 240$ to 260 heartbeats, i.e., $\sim 4$ to $5 \mathrm{~min}$. Run sequence 3 according to Table A11.4.5.

27. Load the images into a flow-quantification software program, draw a region of interest along the inside border of the aorta and let the program calculate the flow through the region-of-interest. Make sure that the velocity encoding sensitivity was correct to avoid aliasing in the graph of flow versus cardiac phase. Repeat the measurement, if necessary, with a larger velocity encoding range.

As a result of the image acquisition, two sets of images should be obtained: images showing the signal magnitude, and images showing the signal phase. Anatomical structures can be easily identified on the magnitude images, while the phase images are used for the flow velocity estimate.

A velocity encoding (Venc) sensitivity of $150 \mathrm{~cm} / \mathrm{sec}$ is recommended for the initial measurement. In the presence of a stenosis, peak flow velocities will significantly exceed this range and require an increase of the Venc range from $150 \mathrm{~cm} / \mathrm{sec}$ to somewhere $\sim 300$ $\mathrm{cm} / \mathrm{sec}$, depending on stenosis severity. Velocities outside the Venc range will appear on the graph as discontinuities, i.e., velocities outside the Venc range are folded back or aliased into the Venc range.
Acquired Heart

Disease

A11.4.11 
Table A11.4.6 Primary Clinical Imaging Parameters for Sequence 4 (Real-Time Cine Sequence)

\begin{tabular}{|c|c|}
\hline Patient position & Supine \\
\hline Scan type & $\begin{array}{l}\text { Single-shot, steady-state free } \\
\text { precession sequence }\end{array}$ \\
\hline Imaging plane (orientation) & Double oblique, short-axis view \\
\hline Central slice or volume center & Mid-level of left ventricle \\
\hline Echo time $\left(T_{\mathrm{E}}\right)$ & $1.15 \mathrm{msec}$ \\
\hline Receiver bandwidth (RBW) & 179 kHz (1398 Hz/pixel) \\
\hline Repeat time $\left(T_{\mathrm{R}}\right)$ & $1.4 \mathrm{msec}$ \\
\hline Delay time $\left(T_{\mathrm{D}}\right)$ & $0 \mathrm{msec}$ \\
\hline Flip angle (FA) & $50^{\circ}$ \\
\hline Fields of view $\left(\mathrm{FOV}_{\mathrm{x}}, \mathrm{FOV}_{\mathrm{y}}\right)$ & $\begin{array}{l}300 \mathrm{~mm}, 300 r \mathrm{~mm}, r=3 / 4 \\
\text { (rectangular field of view) depending } \\
\text { on body habitus }\end{array}$ \\
\hline Resolution $(\Delta x, \Delta y)$ & $2.34 \mathrm{~mm}, 4.41 \mathrm{~mm}$ \\
\hline Number of data points collected $\left(N_{\mathrm{x}}, N_{\mathrm{y}}\right)$ & $\begin{array}{l}128,68 r \text {, with } r=3 / 4 \text { (rectangular } \\
\text { field of view) depending on body } \\
\text { habitus }\end{array}$ \\
\hline Display matrix $\left(D_{\mathrm{x}}, D_{\mathrm{y}}\right)$ & 128,128 \\
\hline Slice thickness $(\Delta z)$ & $10 \mathrm{~mm}$ \\
\hline Number of slices & 1 \\
\hline Slice gap & N/A \\
\hline Number of acquisitions $\left(N_{\mathrm{acq}}\right)$ & 1 \\
\hline Swap read and phase encoding & Swap if wrap-around is reduced \\
\hline Saturation pulses & No \\
\hline Number of cardiac phases & $(R$-to- $R$ interval $) \times 150 \% /\left(T_{\mathrm{R}} \times N_{\mathrm{y}}\right)$ \\
\hline ECG gating & No \\
\hline Scan time & 2 heartbeats \\
\hline
\end{tabular}

\section{Sequence 4: Real-time cine sequence}

In the absence of a regular heart rhythm, ECG-triggering of a cine acquisition will yield unsatisfactory results. In this situation, a very rapid imaging sequence allowing real-time acquisition may be used (Setser et al., 2000). Table A11.4.6 provides the details for very fast steady-state free precession (SSFP) imaging that meets the necessary requirements.

28. Load sequence 3 (based on parameters in Table A11.4.6) and disable ECG triggering. Trim the field of view to the smallest possible size. Due to the reduced number of phase-encoding steps the spatial resolution in the phase-encoding direction will be too low unless the $\mathrm{FOV}_{y}$ is on the order of $300 \mathrm{~mm}$. Use a rectangular field of view if possible.

The resolution in the phase-encoding direction should be below $4 \mathrm{~mm}$.

29. Instruct the patient to breathe normally. Set the number of images (or number of cardiac phases) to be acquired such that they cover a couple of heart intervals. Run sequence 4 according to the parameters given in Table A11.4.6.

The temporal resolution is on the order of 70 msec with $\sim 50$ phase-encoding steps $\left(N_{y}\right)$.

Cardiac Function

Evaluation with Cine MRI of the

Heart 


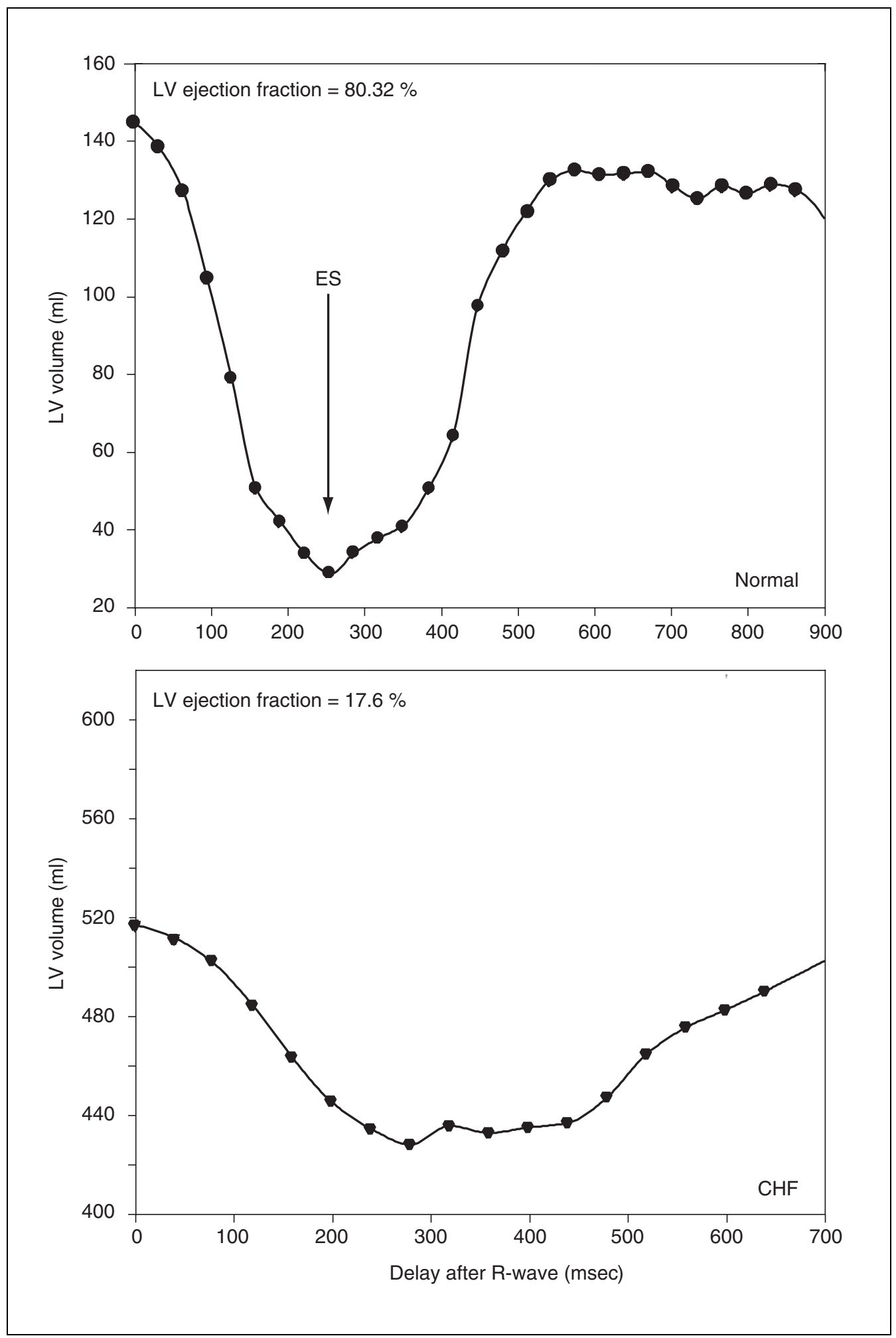

Figure A11.4.6 Example of variation of left ventricular volume over the cardiac cycle for $(\mathbf{A})$ a healthy volunteer, and (B) a patient with congestive heart failure (CHF). The CHF patient had an enlarged ventricle (i.e., large volume), and a very low ejection fraction (EF). Because of the low EF, the curve in (B) is relatively flat. Ventricular volumes were calculated by Simpson's rule from a set of short-axis images. The endocardial border had been traced on each cine frame to obtain a complete LV volume versus time curve.

Acquired Heart

Disease

A11.4.13 


\section{Post-processing}

For determination of the ventricular volumes, ejection fraction, and regional wall motion, the cine images need to be loaded into a program for image segmentation, i.e., the endocardial and epicardial borders need to be identified at least on the end-diastolic (ED) and end-systolic (ES) frames of the cine loops.

31. As a first step, select cine loops that provide coverage from base to apex and load them into the analysis software.

The program will "blindly" calculate chamber volumes based on the slice positions for which data have been loaded, which leads to incorrect results if the slice coverage is insufficient. For cine loops in the short axis view, one should include at the base of the heart only those cardiac phases of the cardiac cycle where the endocardial volume falls below the valve plane. This means that one excludes those frames in the cine loop from the calculation of volumes where the slice position is in or above the valve plane. Generally, such exclusion is performed by default for those frames where the user did not draw endocardial and epicardial borders.

32. If all images in a cine loop are segmented, then obtain a curve showing the variation of the ventricular chamber volume over the cardiac cycle. If EDV and ESV denote, end-diastolic and end-systolic volumes, respectively, use the formula (EDVESV)/EDV for calculation of the ejection fraction, with the result expressed in percent.

Figure A11.4.6 shows two examples of left ventricular volume versus time curves for a normal volunteer and a patient with congestive heart failure $(\mathrm{CHF})$, respectively. In the CHF patient, the ejection fraction is very low, and the chamber volumes are significantly enlarged, both signs of a failing heart.

All global function parameters can be derived from such a volume versus time curve. The analysis of the images can be reduced to segmentation of ED and ES frames if only parameters such as ejection fractions are needed, as they only require ED and ES volumes. Identification of the ES frame in a cine loop is generally straightforward but there are rare instances where dyskinesis can lead to enlargement of a portion of the ventricle during systole.

\section{COMMENTARY}

\section{Background Information}

MRI is considered to be the gold standard for calculating hemodynamic parameters of cardiac function such as ejection fraction (EF), end-diastolic volume (EDV), end-systolic volume (ESV), stroke volume (SV), and cardiac mass. While this accuracy (Matheijssen et al., 1996) is not often needed for clinical patient management, it is not uncommon that a patient has poor acoustic windows for echocardiography. Gated blood pool nuclear medicine scans can also provide hemodynamic data, but performs poorly in the right ventricle and cannot provide the richness of anatomical and functional information of MRI. Thus, MRI is a stand-alone examination or a cardiac MRI provides additional information for some other purpose.

The contrast between the myocardium and the blood pool is of crucial importance for delineation of the endocardial border and determination of ventricular volumes. Myocardial-blood pool contrast can be improved with the use of contrast agents. These may be extracellular agents such as Gd-DTPA (Pennell et al., 1993), however, blood pool agents, such as iron oxide ultra-small particles (USPIO), or albumin-binding, gadolinium-based contrast agents, appear to be better suited for this purpose (Stillman et al., 1997). On scanners with high-performance gradients, true FISP (fast imaging with steady state free precession) provides excellent myocardial-blood pool contrast (Barkhausen et al., 2001; Plein et al., 2001; Thiele et al., 2001). It is, however, sensitive to off-resonance effects and turbulence. It is critical to have consistent breath holding. Generally, the most reproducible diaphragm position is obtainable at end expiration. Oxygen by a nasal cannula can be used to improve the
Cardiac Function

Evaluation with

Cine MRI of the

Heart

A11.4.14

Supplement 10 
breath-hold duration. A few minutes of instruction can also improve reproducibility. For noncooperative patients, it is best to permit free quiet breathing and obtain an average over several acquisitions to minimize respiratory artifact. A respiratory navigator can be helpful if available.

When time is of the essence, a simple biplane method may be used to calculate left ventricular volumes (Cranney et al., 1990). This assumes an ellipsoidal model for the left ventricle that is reasonably true for normal hearts, but becomes less valid as the shape of the heart changes as seen with myocardial infarction. Both two-chamber and four-chamber views are obtained and the area bounded by the endocardial borders is obtained at end diastole (assumed to be represented by the first cardiac phase) and end systole. The volume is calculated as:

$$
\text { volume }=\left(8 A_{2} A_{4}\right) /\left(3 \pi L_{\text {min }}\right)
$$

where $A_{2}$ and $A_{4}$ are the two-chamber and fourchamber areas, respectively, and $L_{\min }$ is the length of the shorter long axis. Geometric models for the right ventricle have also been developed, although less fully evaluated (Czegledy and Katz, 1993).

A more robust and accurate method uses a series of short axis images from the base of the heart to the apex. This method assumes no geometric models that may be invalid in the presence of disease. Volumes are determined by calculating the area bounded by the endocardium in each section. These are added and multiplied by the slice thickness to calculate the volume (Simpson's rule method). Because of through-plane motion, it is not uncommon for all, or part, of a systolic phase image to contain the atrium while the end-diastolic phase image represents the ventricle. Since the base of the heart contributes so greatly to the overall volume, it is critical to measure the entire ventricle without including the atrium. Normally, the ventricle thickens during systole whereas the atrium remains thin. This can be used as a guide to include only that portion that thickens in the volume measurement.

The effective ejection fraction is decreased in the presence of either mitral or aortic regurgitation. The effective ejection fraction may be calculated by the use of a velocity map of the ascending aorta. This is obtained in a cross section at the level of the right pulmonary artery taking care to avoid areas with sternal wire or clip artifact. For the determination of aortic valve regurgitation, it was found that the slice position is critical with best accuracy when it falls approximately between the aortic valve and the coronary ostia (Chatzimavroudis et al., 1997). Pixel-phase intensity is proportional to the velocity of blood flow. Generally, a value of velocity encoding of $150 \mathrm{~cm} / \mathrm{sec}$ is adequate unless there is significant aortic stenosis. A nonsegmented pulse sequence is preferred so that there is optimal temporal resolution. The mean flow is calculated as the integral over the cross-sectional area $(S)$ for the velocity $(v)$ as:

$$
\bar{q}=\oint \vec{v} \cdot d \vec{s}
$$

The effective (forward) stroke volume then may be calculated as:

$$
S V_{\text {effective }}=\int_{0}^{R-\text { to }-R \text { interval }} \bar{q} \mathrm{~d} t
$$

where the time integral is over the cardiac cycle (one $R$-wave to the next). This is more similar to the value that would be obtained from thermodilution methods than from the cine data in that valvular incompetence is implicitly accounted for. For simplicity, an assumption is made that there is only mitral regurgitation (isolated aortic insufficiency can be similarly defined). The mitral regurgitant volume $\left(V_{\mathrm{MR}}\right)$ is

$$
\begin{aligned}
& V_{\mathrm{MR}}=\mathrm{SV}_{\text {total }}-\mathrm{SV}_{\text {effective }}=(\mathrm{EDV}- \\
& \mathrm{ESV})-\mathrm{SV}_{\text {effective }}
\end{aligned}
$$

Where the total stroke volume, $\mathrm{SV}_{\text {total }}$ can be calculated from the short axis cine set as described in the Post-Processing section. Alternatively, if only two-chamber and four-chamber views have been acquired, then the above "biplane" formula can be used to calculate ESV and EDV. The mitral regurgitant fraction (MR) is calculated as

$$
\mathrm{MR}=V_{\mathrm{MR}} / \mathrm{SV}_{\text {total }}
$$

and the effective ejection fraction ( $\left.\mathrm{EF}_{\text {effective }}\right)$ as

$$
\mathrm{EF}_{\text {effective }}=\mathrm{SV}_{\text {effective }} / \mathrm{EDV} \text {. }
$$

While this may seem complicated, the net forward- and reverse-flow volumes over a cardiac cycle are provided by standard evaluation software so the remaining calculations are straightforward.

The combination of both aortic and mitral regurgitation limits the accuracy of the calculated respective regurgitant volumes, as does the presence of an intracardiac shunt. Nevertheless, the effective EF should be a reliable number. In this case, one can assume that systole is defined as the period of forward flow and
Acquired Heart

Disease

A11.4.15 
that the mitral valve should be closed. There is no aortic regurgitation during systole. The difference between $\mathrm{SV}_{\text {total }}$ and the forward-flow volume is then taken to be $V_{\mathrm{MR}}$.

Occasionally, $V_{\mathrm{MR}}$ can be negative. This occurs when there are dyskinetic segments or dyssychrony of left ventricular function so that an ESV is difficult to define. In such a case, it is not possible to calculate $V_{\mathrm{MR}}$ by this method. The calculation of $\mathrm{EF}_{\text {effective }}$ is still valid and preferred over the use of the ejection fraction calculated solely from the cine data. Reliability of this method depends on a regular heart rhythm and consistent breath holding.

\section{Steady-state free precession imaging}

Acquisition of image data during steadystate free precession method represents one of the most significant recent developments for the assessment of cardiac hemodynamics with MRI (Bundy et al., 1999). A variety of acronyms have been coined by different equipment manufacturers for this pulse sequence type, e.g., trueFISP (Siemens), Fiesta (GE), and Balanced Fast Field Echo (Philips). The essential characteristic of this technique is the goal to avoid any spoiling of transverse magnetization (see UNIT B5.2 and Duerk et al., 1998). In the steady state, a radio frequency excitation pulse in an SSFP sequence rotates a fraction of the longitudinal magnetization into the transverse plane, and at the same time returns the transverse magnetization that was refocused at the end of the previous repeat time $\left(T_{\mathrm{R}}\right)$ to the longitudinal direction. Careful refocusing of the transverse magnetization after slice-selective excitation, phase-encoding, and echo-readout is essential for this technique. In SSFP imaging, the trajectory of the magnetization components is controlled by the radio frequency (RF) pulses and gradient waveforms without relying on longitudinal relaxation between RF pulses and replenishing the longitudinal magnetization. Therefore, saturation by repeated RF excitation is avoided, and the image contrast is dominated by the $T_{2} / T_{1}$ relaxation times. With SSFP imaging, one can theoretically apply $90^{\circ}$ radio frequency pulses instead of small flip angle pulses, which produces a significantly higher signal-to-noise ratio in the images than with conventional gradient echo images. For reasons of RF deposition and contrast, smaller flip angles close to $45^{\circ}$ are more appropriate.

In conventional gradient echo imaging with prospective triggering, the pulse sequence is suspended once the last $k$-space line of the phase-encoding steps has been collected. The sequence resumes once an $R$-wave peak has been detected on the ECG. By contrast, with steady-state free precession sequences, the sequence runs continuously until the end of the entire image acquisition to avoid any disturbance to the steady-state equilibrium. In SSFP imaging, the preservation of coherence of the transverse magnetization is still limited by the $T_{2}{ }^{*}$ decay. Any phase errors introduced, e.g., by off-resonance shifts, are accumulated over the image acquisition. SSFP imaging is, therefore, more sensitive than conventional gradient-echo imaging to off-resonance shift and magnetic-field homogeneities. Homogeneous magnetic fields by shimming are necessary to obtain good results, much more so than with conventional gradient echo imaging.

SSFP imaging appears to produce the most striking improvements in image quality by comparison to conventional gradient echo imaging when studies are performed on patients with compromised cardiac function, e.g., in patients with heart failure. In conventional gradient echo cine MRI, the contrast between myocardium and blood pool is, to a large extent, the result of differences in magnetization saturation in myocardial tissue and blood flowing in and out of the imaging slice. With compromised pumping function, the inflow of unsaturated magnetization is reduced and this results in a corresponding loss of contrast between myocardium and blood pool. Figure A11.4.7 shows an example in the same patient of conventional gradient echo images and SSFP images. The differences in contrast between conventional gradient echo images and SSFP images are most pronounced for long-axis views, as the bright-blood effect is reduced when the predominant direction of flow is parallel to the slice plane (long-axis view), instead of perpendicular to the image plane (short-axis view). SSFP sequences use relatively short echo times which may in some cases cause an observer to miss a jet, or overemphasize the jet. Standard gradient echo sequences, which use a longer $T_{\mathrm{E}}$ than SSFP sequences, generally allow better visibility of dephasing. Thus, jets caused by valvular disease or intracardiac shunts are better seen using standard gradient echo sequences (Pereles et al., 2001).

\section{Post-processing software}

Currently available software packages impose the requirement that the heart be covered
Eardiac Function
Evaluation with
Cine MRI of the
Heart

A11.4.16 
A

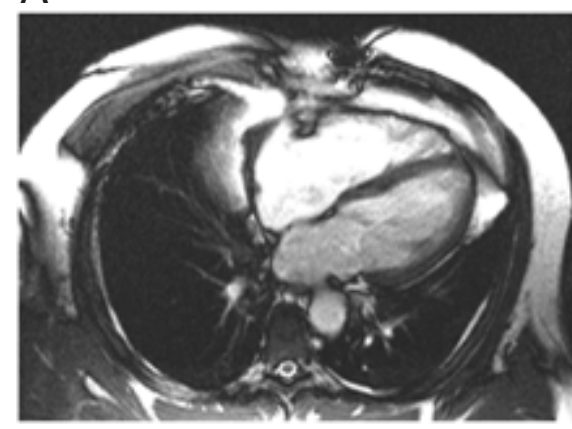

C

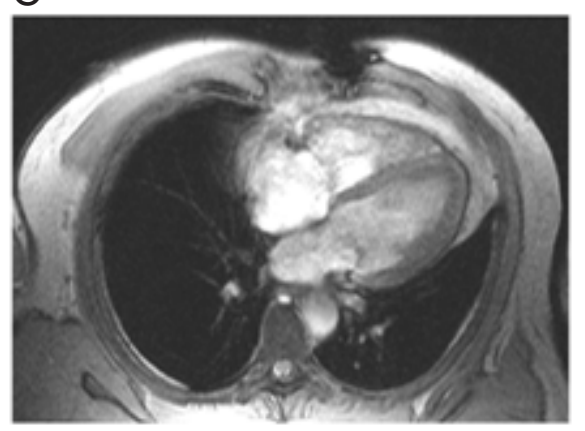

B

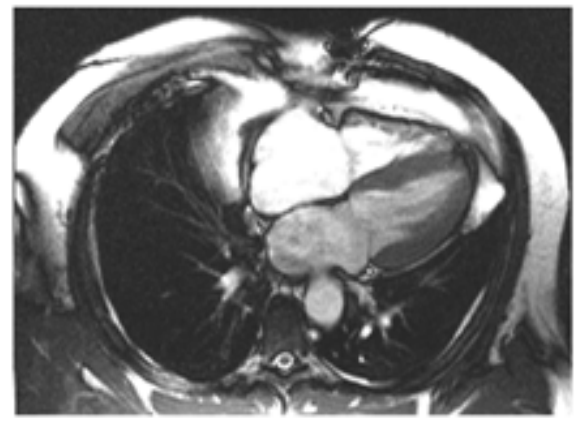

$\mathrm{D}$

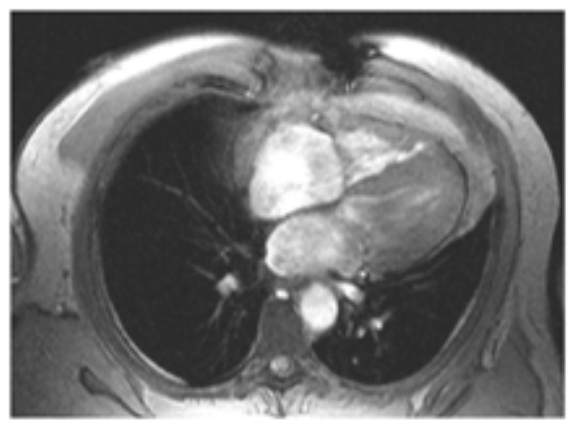

Figure A11.4.7 Comparison of cine acquisition with steady-steady free precession (SSFP) technique (A, B); with "conventional" gradient-echo sequence (C, D). The SSFP technique affords better contrast between blood pool and myocardium. Poorer contrast in the cases of (C) and (D) can lead to an underestimate of ventricular volume and an overestimate of myocardial mass.

with parallel slices from base to apex. Volumes and myocardial mass are calculated with Simpson's rule. This yields good accuracy but requires a relatively large number of acquisitions. Several long-axis views of the heart, rotated around a central axis could, in principle, yield similar accuracy, but would require a more complex reconstruction of the ventricular shape. Dulce et al. (1993) already showed that a combination of two long-axis views and one to two short-axis views yield results in close agreement with a significantly larger number of co-planar short-axis views (Dulce et al., 1993). Despite these encouraging results, the current software packages generally cannot accommodate more flexible acquisition strategies with multiple rotated and shifted image planes (Bloomgarden et al., 1997).

\section{Critical Parameters and Troubleshooting}

\section{ECG tracings}

Cardiac motion artifacts in the images can generally be traced to either unreliable gating of the sequence or the inability of the patient to suspend breathing motion during image acquisition. Irregular gating of the pulse sequence results in an irregular noise pattern from the switching gradients, and on the images, the heart appears blurred. Unreliable gating with ECG can often be remedied by switching the order of the ECG leads (electronically) or, as a last resort, by repositioning the leads on the chest. With an image acquisition triggered by the $R$-wave of the ECG, the first frame of the cine loop coincides with the beginning of the isovolumetric contraction.

\section{Specific absorption rate with steady-state free precession imaging}

A potential disadvantage of SSFP imaging is the radio frequency power deposition due to high flip-angle pulses. The software on the scanner limiting the tissue absorption of radiofrequency power may prevent a pulse sequence from running. The user then has to reduce the flip angle, and in practice, this means that the SSFP sequences are used with flip angles considerably $<90^{\circ}$, and more on the order of $40^{\circ}$ to $60^{\circ}$.
Acquired Heart

Disease

A11.4.17 


\section{Motion and flow artifacts}

Breathing motion during image acquisitions that require breath-holding of the patient results in ghost images replicated along the phase-encoding direction. Ghosting will be most noticeable from regions that produce very high signal intensities such as subcutaneous fat. If the patient is not able to hold his or her breath, then more than one acquisition should be acquired to average out the breathing motion, although breathing motion will still cause blurring of the endocardial and epicardial borders.

Bright-blood gradient echo imaging has traditionally employed flow-compensated gradient waveforms to reduce signal loss due to flow. By switching off flow compensation, one can substantially reduce the echo time, and this can be used as an advantage to speed up the image acquisition without producing a significant reduction in image quality for typical rest heart rates ( $<80$ beats per minute) if the echo time is on the order of 2 to $3 \mathrm{msec}$. Alternatively, one can put the $T_{\mathrm{E}}$ reduction to use by reducing the $T_{\mathrm{R}}$, and thereby improve the temporal resolution, or acquire multiple slices during each breath-hold.

SSFP sequences are more sensitive to flow and motion than conventional FLASH (fast low angle shot) cine MRI. The initial experiences with this technique suggest that the advantages of SSFP over conventional gradient echo-imaging may be partially lost at heart rates above $\sim 130$ to 140 beats per minute. At high heart rates, as encountered during inotropic stimulation with dobutamine, the user may consider switching back to conventional gradient echo imaging if the image quality is unsatisfactory.

\section{Overestimation of stroke volume}

Although a "stack" of cine loops in the short-axis view for slice positions from base to apex are sufficient for the evaluation of ventricular function, one should not skip over the acquisition of long-axis cine loops. A qualitative evaluation of aortic insufficiency can be performed in the long-axis view, by assessing the width and depth of the jet. Cardiac cine should be augmented by phase-contrast measurements for a more precise assessment of the forward stroke volume and the degree of aortic insufficiency.

\section{Anticipated Results}

Lorenz et al. (1999) reported normal ranges of left and right ventricular mass and ventricular volumes measured with cine magnetic resonance imaging from a study involving 75 healthy subjects. This report, and others that may appear in the literature with the more widespread adoption of the cine MRI, will constitute a valuable database against which to evaluate cardiac pathologies.

Systematic comparisons of ventricular volumes calculated from conventional gradient echo images and SSFP images show that SSFP imaging gives higher values for the cavity volumes and smaller values for myocardial mass. The most accepted explanation for this discrepancy is that stasis near the ventricular wall will result on conventional gradient echo images in reduced signal intensity near the endocardial border that is easily mistaken for a myocardial signal. In areas with pronounced trabeculations, the reduced brightness of the signal will create a bias towards extending the endocardial border into the blood pool. On high quality SSFP images, one can easily identify areas of the myocardial wall with extensive endocardial trabeculae, something that is not easily recognizable with bright-blood gradient echo imaging. For segmentation of the images, the exact results for ventricular volumes will depend on whether the contours are drawn through the "middle" of the trabeculae, or whether they are excluded. For follow-up studies, as in therapy trials, it becomes important to stick to one and the same prescription for image segmentation.

The use of inotropic agents such as dobutamine in combination with echocardiography is quite common to detect wall-motion abnormalities due to ischemia. A comparison of wall motion studies performed with MRI versus ultrasound in an important study by Nagel et al. (1999) has demonstrated a significantly higher diagnostic accuracy of stress MRI in comparison to stress echocardiography. Monitoring patients during dobutamine stress remains an area of concern that has impeded the wider use of MRI for stress wall motion studies. Near real-time feedback to the MRI operator of the cine loops can provide some degree of safety in preventing excessive ischemic insults, a dangerous degradation of global ventricular function, and/or cardiac arrest.

\section{Literature Cited}

Barkhausen, J., Ruehm, S.G., Goyen, M., Buck, T., Laub, G., and Debatin, J.F. 2001. MR evaluation of ventricular function: True fast imaging with steady-state precession versus fast low-angle shot cine MR imaging: Feasibility study. Radiology 219:264-269.

Bloomgarden, D.C., Fayad, Z.A., Ferrari, V.A., Chin, B., Sutton, M.G., and Axel, L. 1997. Global cardiac function using fast breath-hold 
MRI: Validation of new acquisition and analysis techniques. Magn. Reson. Med. 37:683-692.

Bundy, J., Simonetti, O., Laub, G., and Finn, J.P. 1999. Segmented trueFISP cine imaging of the heart. Seventh Meeting of the International Society for Magnetic Resonance in Medicine. Philadelphia.

Chatzimavroudis, G.P., Walker, P.G., Oshinski, J.N., Franch, R.H., Pettigrew, R.I., and Yoganathan, A.P. 1997. The importance of slice location on the accuracy of aortic regurgitation measurements with magnetic resonance phase velocity mapping. Ann. Biomed. Eng. 25:644-652.

Cranney, G.B., Lotan, C.S., Dean, L., Baxley, W., Bouchard, A., and Pohost, G.M. 1990. Left ventricular volume measurement using cardiac axis nuclear magnetic resonance imaging. Validation by calibrated ventricular angiography. Circulation 82:154-163.

Czegledy, F.P. and Katz, J. 1993. A new geometric description of the right ventricle. J. Biomed. Eng. 15:387-391.

Duerk, J.L., Lewin, J.S., Wendt, M., and Petersilge, C. 1998. Remember true FISP? A high SNR, near 1 -second imaging method for $T_{2}$ - like contrast in interventional MRI at .2 T. J. Magn. Reson. Imaging 8:203-208.

Dulce, M.C., Mostbeck, G.H., Friese, K.K., Caputo, G.R., and Higgins, C.B. 1993. Quantification of the left ventricular volumes and function with cine MR imaging: Comparison of geometric models with three-dimensional data. Radiology 188:371-376.

Lorenz, C.H., Walker, E.S., Morgan, V.L., Klein, S.S., and Graham, T.P. Jr. 1999. Normal human right and left ventricular mass, systolic function, and gender differences by cine magnetic resonance imaging. J. Cardiovasc. Magn. Reson. $1: 7-21$.

Matheijssen, N.A., Baur, L.H., Reiber, J.H., van der Velde, E.A., van Dijkman, P.R., van der Geest, R.J., de Roos, A., and van der Wall, E.E. 1996. Assessment of left ventricular volume and mass by cine magnetic resonance imaging in patients with anterior myocardial infarction intra-observer and inter-observer variability on contour detection. Int. J. Card. Imaging 12:11-19.

Nagel, E., Lehmkuhl, H.B., Bocksch, W., Klein, C., Vogel, U., Frantz, E., Ellmer, A., Dreysse, S., and Fleck, E. 1999. Noninvasive diagnosis of ischemia-induced wall motion abnormalities with the use of high-dose dobutamine stress MRI: Comparison with dobutamine stress echocardiography. Circulation 99:763-770.

Pennell, D.J., Underwood, S.R., and Longmore, D.B. 1993. Improved cine MR imaging of left ventricular wall motion with gadopentetate dimeglumine. J. Magn. Reson. Imaging 3:13-19.
Pereles, F.S., Kapoor, V., Carr, J.C., Simonetti, O.P., Krupinski, E.A., Baskaran, V., and Finn JP. 2001. Usefulness of segmented trueFISP cardiac pulse sequence in evaluation of congenital and acquired adult cardiac abnormalities. Am. J. Roentgenol. 177:1155-1160.

Plein, S., Bloomer, T.N., Ridgway, J.P., Jones, T.R., Bainbridge, G.J., and Sivananthan, M.U. 2001. Steady-state free precession magnetic resonance imaging of the heart: Comparison with segmented $k$-space gradient-echo imaging. J. Magn. Reson Imaging 14:230-236.

Schulen, V., Schick, F., Loichat, J., Helber, U., Huppert, P.E., Laub, G., and Claussen, C.D. 1996. Evaluation of $k$-space segmented cine sequences for fast functional cardiac imaging. Invest. $R a$ diol. 31:512-522.

Setser, R.M., Fischer, S.E., and Lorenz, C.H. 2000. Quantification of left ventricular function with magnetic resonance images acquired in real time. J. Magn. Reson. Imaging 12:430-438.

Shellock, F.G. 1996. Pocket Guide to MR Procedures and Metallic Objects. Lippincott-Raven, Philadelphia.

Stillman, A.E., Wilke, N., and Jerosch-Herold, M. 1997. Use of an intravascular $T_{1}$ contrast agent to improve MR cine myocardial-blood pool definition in man. J. Mag. Reson. Imaging 7:765767.

Thiele, H., Nagel, E., Paetsch, I., Schnackenburg, B., Bornstedt, A., Kouwenhoven, M., Wahl, A., Schuler, G., and Fleck, E. 2001. Functional cardiac MR imaging with steady-state free precession (SSFP) significantly improves endocardial border delineation without contrast agents. $J$. Magn. Reson. Imaging 14:362-367.

\section{Internet Resources}

http://www.medis.nl

The software package MASS (Magnetic Resonance Analytical Software System) has been developed for the quantitative analysis of multi-slice/multi-cardiac phase left and right ventricular function.

http://www.siemensmedical.com

Leonardo is an analysis software package from Siemens Medical Systems for evaluating cardiac magnetic resonance cine images and assessment of ventricular functions.

Contributed by Arthur E. Stillman and Michael Jerosch-Herold

University of Minnesota

Minneapolis, Minnesota
Acquired Heart

Disease

A11.4.19 